\title{
3 Research Square

\section{Formation Mechanism of Well-Ordered Densely Packed Nanoparticle Superlattices Deposited from Gas Phase on Template-Free Surfaces}

\section{Chang Liu}

Nanjing University

Fei Liu

Nanjing University

Chen Jin

Nanjing University

Sishi Zhang

Nanjing University

Lianhua Zhang

Nanjing University

Min Han ( $\nabla$ sjhanmin@nju.edu.cn )

Nanjing University https://orcid.org/0000-0002-8911-8018

\section{Research Article}

Keywords: ordered nanocluster monolayer, gas phase cluster deposition, self-assembling, densely-packed, surface migration, attractive interaction

Posted Date: July 16th, 2021

DOl: https://doi.org/10.21203/rs.3.rs-706708/v1

License: (c) (i) This work is licensed under a Creative Commons Attribution 4.0 International License.

Read Full License

Version of Record: A version of this preprint was published at Nanoscale Research Letters on November 30th, 2021. See the published version at https://doi.org/10.1186/s11671-021-03635-7. 


\section{Abstract}

Superlattices of nanoparticles are generally produced based on solution chemistry processes. In this paper, we demonstrate that self-assembled monolayer structures of nanoparticles with superlattice periodicities can also be produced on template-free surfaces in the gas phase cluster beam deposition process. It is found the packing of Fe nanoparticles corresponds to an average two-dimensional denselypacked lattice with a hexagonal summary. By controlling the nanoparticle coverage, the two-dimensional densely-packed monolayer morphology can spread to the whole substrate surface being deposited. A formation mechanism of the ordered monolayers is proposed by considering the balance between the diffusion rate of the nanoparticles and their filling speed on the substrate surface determined by the deposition rate, and the ordering driven by the inter-particle attractive forces. The model is strongly supported by a serial of carefully designed cluster deposition experiments.

\section{Introduction}

Nanoparticle superlattices, in which the particles behave as artificial atoms and are arranged with crystallographic orders, have attracted tremendous attention since they were first reported [1]. Superstructures assembled from tailored nanoparticle building blocks enable the design of novel materials and optimizing, and/or tuning the properties and performance of the nanomaterials [2-7]. As an elegant alternative to the lithographic approaches, nonlithographic bottom-up approaches based on thermodynamically driven self-organization processes are especially appealing, because of their advantages such as simpler technology and potential for large-scale production of very small structures at scales beyond the current limits of lithographic techniques.

Generally, ordered arrays or superlattices of nanoparticles are produced based on solution chemistry processes. Nanoparticles from a colloidal solution can form ordered arrays upon spontaneous organization of monodispersed nanoparticles encapsulated in surfactant monolayers [8-10], or by using biological molecules and their specific interactions $[11,12]$. Typically, the nanostructure assembled from colloidal solution has organic monolayers to encapsulate the particles, which produces a soft structure. Furthermore, such chemical additive often facilitates tailoring the intrinsic property of the nanoparticle assembly and sometimes becomes a significant limitation [13].

In recent years, efforts were also devoted to fabricate nanoparticle arrays with predeterminate patterns from gas phase. Unlike the nanostructures from a colloidal solution, nanoparticle assemblies formed in the gas phase do not have organic surface encapsulates, so that pure inter-particle interfaces and intrinsic properties can be expected. However, to fabricate nanoparticle assemblies with controlled spatial organizations, various templates with pre-patterned surface features have to be used. By performing gas phase atom deposition, ordered arrays of uniform nanoparticles were fabricated via strain-induced spontaneous nucleation of three-dimensional nanometer-sized islands of on top of a strained epilayer $[14,15]$. Arrays of quasi-one-dimensional Ag nanoparticle chains were generated by trapping the gas phase deposited nanoparticles at the step edges of graphite surface [16]. Shi et al. used self-assembled 
nano-patterns of block copolymer as templates for gas phase cluster deposition to produce twodimensional (2D) arrays of metal nanoparticles [17]. Although selective decoration of phase-separated diblock copolymer template [18] with vacuum-deposited metals has become a common way to fabricate patterned nanoparticle arrays, the assembly such produced contained many defects and the packing of the nanoparticles was not so compact due to the relatively large spatial period in the self-assembled patterns of the block copolymer templates. Up to now, it is unsuccessful to assemble ordered arrays of nanoparticles via gas phase deposition on template-free surface.

Gas-phase nanocluster deposition provides a well-developed process able to produce nanoparticle-based nanostructures with a high level of control on size, density and functional assembling morphology [1921]. Generally, nanoparticles deposited at low kinetic energy are able to diffuse on the surface with a high mobility and tend to aggregate [22]. On the other hand, the migrations of the nanoparticles are constrained by the surface defects. Therefore, in previous studies, either random arrays [23] or large ramified aggregates [24] of nanoparticles were obtained with a moderate deposition mass, depending on the impact energy of the nanoparticles and the substrate. It was commonly believed such structures are strongly disordered with neither long-range orders nor short-range orders.

In this paper, we demonstrate that well-ordered densely-packed nanoparticle monolayers could be formed on the smooth surface of amorphous carbon substrate by performing gas phase cluster deposition without any preformed template. With a moderate coverage, ordered arrays of densely packed nanoparticles are observed in the submicron length scale. The nanoparticle monolayers display a certain superlattice periodicity. We carry out a serial of cluster deposition experiments to analyze the formation mechanism of the nanoparticle superlattice morphology, by considering a balance among various factors of cluster deposition dynamics, such as the flux and the kinetic energy of the clusters, as well as the migration ability of clusters on the substrate surface.

\section{Experimental Section}

\section{Deposition of nanoparticle arrays}

Fe and TiN nanoparticles were generated by using a magnetron plasma gas aggregation cluster source $[23,25]$ consisting of a liquid nitrogen cooled aggregation tube ended with an orifice $3 \mathrm{~mm}$ in diameter. A schematic drawing depicting this setup is presented in Fig. 1. The magnetron discharge was operated at a pressure of $64 \mathrm{~Pa}$ in argon stream. Atoms were sputtered from the target on the magnetron discharge head and aggregated into clusters in the argon stream. An aggregation length (the length of the space where cluster formation took place) of $75 \mathrm{~mm}$ was used. The clusters were swept by the argon gas stream out of the aggregation tube into a high vacuum $\left(<1 \times 10^{4} \mathrm{~Pa}\right)$ chamber through the orifice and formed a collimated nanoparticle beam with a divergence of about $3^{\circ}$. The nanoparticles were deposited on the substrates fixed on a rotary sample holder in the high vacuum chamber. The incident angle of the nanoparticles on the substrate could be tuned between $0^{\circ}$ to $90^{\circ}$ by rotating the sample holder. The 
deposition rate was monitored with a quartz crystal microbalance and controlled precisely by the discharge power applied to the cathode with a DC power supply (MDX500, Advanced Energy).

\section{Characterizations of the nanoparticle arrays}

The nanoparticles were deposited on ultrathin amorphous carbon films on formvar-coated 300 mesh copper grids. The structure and morphology of the deposited nanoparticle films were characterized with a transmission electron microscope (TEM, FEI TECNAI F20s TWIN) operated at $200 \mathrm{kV}$. The composition of the nanoparticles were analysed using Energy Dispersive X-ray spectroscopy (EDX). To analyze the oxidation state of the Fe nanoparticle arrays, $x$-ray photoelectron spectroscopy (XPS) was carried out with an ESCALABMK-II spectrometer using a monochromatic Mg Ka source.

Fe nanoparticles were also deposited on silicon wafers for magnetization measurements, which were performed by using a superconducting quantum interference device magnetometer (SQUID, MPMS-3).

\section{Results And Discussion}

\section{Analysis of the structure of the nanoparticle film}

Figure 2a shows the TEM image of Fe nanoparticle arrays prepared by depositing the nanoparticle beam vertically on the surface of the amorphous carbon film with a deposition rate of $0.1 \AA \cdot \mathrm{s}^{-1}$. The nanoparticle array is composed of densely-packed 2D monolayer domains ordered over $100-200 \mathrm{~nm}$ scale. A high magnification TEM image of a well-ordered defect-free monolayer domain is shown in Fig. 2b. Typically, the nanoparticle monolayers may contain some defects, such as lattice distortions, dislocations, vacancies or voids, as well as size variations of the nanoparticles. The average nanoparticle size is $6.1 \pm 1.6 \mathrm{~nm}$, as determined using a minimum of 300 nanoparticles in the arrays (Fig. S1). The size dispersion is significantly larger than those in the self-assembled superlattices of thiol-passivated nanoparticles [26, 27]. A high-resolution transmission electron microscopy (HRTEM) image given in Fig. $2 d$ shows the individual Fe nanoparticles are mainly single crystals with spherical shapes. They are randomly oriented on the substrate surface. Prior to the observation, the nanoparticles have been exposed to air for a significantly long time so that their surfaces are sufficiently oxidized, as can be distinguished in the HRTEM image. The existence of the oxidation layer on the nanoparticle surface can be further confirmed by EDX and XPS. As shown in Fig. S2, 0 elements are always observed together with the Fe nanoparticles in the EDX elemental mapping images. XPS measurements also show the evidence of oxidation of Fe nanoparticles. As shown in Fig. S3, both metallic Fe and Fe oxide can be distinguished from the photoemission data of the Fe $2 p$ core levels. After the nanoparticle specimens are cleaned with Ar ion sputtering, the XPS peaks corresponding to the $2 \mathrm{p}$ core levels of pure Fe are greatly enhanced, indicating that Fe oxides only present on the nanoparticle surfaces. Therefore, the nanoparticle arrays can be looked as a compact packing of closely contacted core/shell nanoparticles. The crystalline metal cores are separated from each other with amorphous oxide shells. The oxide shell acts as a passivation layer preventing further oxidation of the Fe nanoparticles. The mean edge-to-edge distance separating 
well-aligned nanoparticles in the closely packed lattice is measured at $1.7 \pm 0.6 \mathrm{~nm}$. Correspondingly, the thickness of the amorphous oxide shell is about $0.85 \mathrm{~nm}$ on average. It should be noted that the oxide shells are formed after the deposition process is finished. Since the nanoparticle deposition is performed under high vacuum condition, the self-assembling occurs in the pure metal nanoparticles, rather than the surface-oxidized nanoparticles. The amorphous oxide shells play no role in the organization of the nanoparticles.

The fast Fourier transform (FFT) of the densely-packed monolayers is shown in Fig. 2c. Well-defined spots arranged in hexagon are shown, attesting to a densely packed nanoparticle lattice ordered over a long range. However, only one hexagon related to the first order is distinguishable, indicating the scale of the ordered monolayer domains is limited.

\section{Tailoring the assembling morphology with nanoparticle deposition conditions}

We have found the deposition rate of the nanoparticles plays a definite role in the formation of the ordered densely packed monolayers. In Fig. 3a-c, TEM images of Fe nanoparticle arrays prepared with deposition rate ranging from $0.3-0.7 \AA \cdot \mathrm{s}^{-1}$ are shown. The FFT of each image is shown as the insets. The deposition time of each specimen is such controlled that a constant nanoparticle coverage (i. e. total deposition mass) on the substrate is maintained. In each image, the average nanoparticle size and distribution (Fig. S1), is almost identical (the average diameter is measured to be $6.0 \pm 1.4 \mathrm{~nm}, 6.1 \pm 1.3 \mathrm{~nm}$ and $6.1 \pm 1.7 \mathrm{~nm}$ respectively). From Fig. $2 \mathrm{a}$ and Fig. 3a-c, we can find that with the increase of the deposition rate, the range scale of the ordered monolayer domains becomes smaller and smaller. We analyse the TEM images by counting the nanoparticle number contained in each monolayer domain. The sizes of the monolayer domains can be compared quantitatively with the nanoparticle numbers they contain. The histograms of the counted nanoparticle numbers are shown in Fig. 3d. The maximum of the distribution tends to smaller nanoparticle numbers with the increase of deposition rate. The average nanoparticle number contained in an individual monolayer domain decreases from 77 at a deposition rate of $0.1 \AA \cdot \mathrm{s}^{-1}$ to 27 at a deposition rate of $0.7 \AA \cdot \mathrm{s}^{-1}$. Meanwhile, the spots present in the FFT pattern become more and more diffuse. With a deposition rate of $0.7 \AA ̊ . \mathrm{s}^{-1}$, only a diffuse ring without any hexagonal symmetry can be seen in the FFT pattern. The nanoparticles in the TEM image display a random distribution on the whole.

In Fig. 3e, the radial distribution functions (RDFs) calculated from the TEM images are shown. For the nanoparticle arrays formed at $0.1 \AA ̊ \cdot \mathrm{s}^{-1}$ and $0.3 \AA \mathrm{As}^{-1}$ deposition rates, the RDF curves display sharp and clear first and second peaks, corresponding to the nearest and subnearest neighbors with average particle-particle intervals of $8 \mathrm{~nm}$ and $17 \mathrm{~nm}$, and a distinguishable third peak corresponding to the third neighbors with an average interval of $24 \mathrm{~nm}$, indicating that the nanoparticle arrays are well-ordered with superlattice periodicities. For the nanoparticle arrays formed at $0.5 \AA \cdot \mathrm{s}^{-1}$ deposition rate, the second peak in the RDF curve becomes much reduced and the third peak is completely indistinguishable, indicating a decreasing organization and reduced lattice periodicity. With a deposition rate of $0.7 \AA \cdot \mathrm{s}^{-1}$, the 
nanoparticle arrays only display a weak first peak in the RDF curve, which strongly reflects the loss of the lattice periodicity and short-range order. It is clear that a low deposition rate is an important parameter dominating the well-ordered monolayer formation.

We have also found the structure of the nanoparticle arrays is correlated with the feature of the substrate surface. Different assembling patterns are obtained with different substrates. Figure 4 shows a TEM image of the Fe nanoparticle arrays deposited on a Formvar film. The deposition is performed with a deposition rate of $0.1 \AA \cdot \mathrm{s}^{-1}$. Although the operation parameter of the cluster source and the deposition mass is identical to that used for the sample shown in Fig. 2, ordered densely-packed morphology could no more be observed. The distribution of the nanoparticles on the surface is completely random. No evidence of organization could be observed. In some area homogeneous coalescences of the nanoparticles form larger particles. It is known the mobility of nanoparticles softly landing on solid surface is strongly dependent on the nature of the surface [20], especially its defect state and binding ability with the deposits. It has been shown that metal nanoparticles have high mobilities on the surface of carbon materials [22, 23], on the contrary, no diffusive aggregation of metal nanoparticles has been observed on the Formvar film surface [28]. Instead, the nanoparticles are mostly pinned where they are deposited. It is difficult for them to diffuse and aggregate on the substrate. Coalescence takes place locally as a fusion process under particle-particle collision in the deposition process. These results suggest that certain mobility is needed once the nanoparticles are deposited on the surface in order to form densely-packed monolayers.

To understand the formation mechanism of the densely-packed nanoparticle monolayers in the gas phase cluster deposition, we have to consider the competition between the diffusion rate of the nanoparticles and the filling speed of the nanoparticles deposited on the substrate surface, which is dependent on the deposition rate. This is similar to the situation that happens in the spontaneous organization process occurring at the liquid/substrate interface to form periodic $2 \mathrm{D}$ arrays of thiolate encapsulated nanoparticles upon solvent evaporation from a droplet of colloidal solution depositing on the substrate. Previously, experiments $[29,30]$ showed that when a droplet of nanoparticle solution was deposited onto a substrate and dried shortly, amorphous nanoparticle aggregates with little uniformity and symmetry were formed. As the droplet was dried more and more slowly, increasing uniformity was observed and finally closely-packed nanoparticle superlattices were formed. With a slow solvent evaporation rate, the nanoparticles benefit from more time to diffuse on the substrate and adjust their sites attached to the nanoparticle assembly, giving rise to a higher level of ordering. Similarly, the nanoparticles deposited on the carbon substrate from gas phase can diffuse on the free surface with a high mobility. If the arrival rate of nanoparticles to the surface is too high, the motion of the nanoparticles on the surface will be limited by each other, and the free area available for each nanoparticle will be soon exhausted. The nanoparticles cannot sufficiently adjust their positions on the surface, resulting in randomly packed aggregates. Moreover, if the sticking coefficient between the nanoparticles remains high, low-density factal aggregates are formed $[22,24]$. However, with a mild deposition rate, the arrival time of the nanoparticles is controlled such that the nanoparticles have enough time to diffuse on the 
free surface and find equilibrium lattice sites on the growing structure. As a result, ordered denselypacked monolayers are formed. As the flux of nanoparticles adding to the surface is increased by increasing the deposition rate, the arrival rate exceeds the surface mobility of nanoparticles and the formation of an inhomogeneous disordered aggregate occurs.

The surface mobility of the nanoparticles is dependent on the interaction between the nanoparticle and the surface. On the surface of organic materials metal nanoparticles are mostly pinned where they are deposited. Although they have high mobility on the perfect surface of carbon substrate, their diffusion may also be limited by the particle diffusion barriers on the surface, such as the defects. If the thermal energy of a nanoparticle is low compared with the binding energy, it may be arrested on the diffusion barriers. It is possible to increase the diffusion length of the nanoparticles either by increasing the temperature or by increasing their lateral migration energies when they land on the surface. Increasing temperature is not preferred in the nanoparticle assembling since it induces sufficient coalescences among nanoparticles, we therefore try to increase the lateral migration energies of the nanoparticles by increasing their momentums along the surface when they impact on the substrate. This is achieved by depositing nanoparticles with glance incidence relative to the substrate surface. Generally, the initial kinetic energy of the nanoparticles generated from a cluster source is several $\mathrm{eV}$ on average. With a glance incidence, a partial of the kinetic energy transfers to the migration energy of the nanoparticle on the surface. This will enhance the abilities of the nanoparticles to escape from the diffusion barriers where they are arrested, so as to increase the migration length of the nanoparticles. In Fig. 5a, a TEM image of the Fe nanoparticle arrays prepared with a $45^{\circ}$ glance incidence angle is shown. The equivalent deposition rate is $0.1 \AA \cdot \mathrm{s}^{-1}$. Comparing with the nanoparticle arrays prepared with same deposition parameters under normal incidence (Fig. 2a), we find the range scale of the ordered monolayer domains is significantly increased, and the hexagonally arranged FFT spots become more sharp, clear and scattered. From the RDF curve shown in Fig. 5b, we can see the subnearest peak is notablely enhanced and sharpened. Especially, the third neighbor peak which is indistinct in the case of the normally deposited samples becomes sharp and clear now, indicating a significant improvement on the organization length and lattice periodicity. It therefore demonstrates a simple way to increase the diffusion length of the nanoparticles so as to realize larger scale ordered monolayers.

It should be noted that the balance between the diffusion rate and the arrival time of the nanoparticles on the surface is not the only condition sufficient for the ordered nanoparticle monolayer formation. The ordering is driven by the interparticle attractive forces. Unlike in the case of self-assembled superlattices of thiolate encapsulated nanoparticles, in which the main contribution to the interaction comes from the surfactant molecules, which produce a soft structure [27], in the present study the interaction dominates the ordered nanoparticle array formation comes from the metal nanoparticles themselves, which produces a rigid hard structure. In the 2D densely-packed monolayer, a nanoparticle falls on the equilibrium site since it receives the maximum attractive interactions from the identical nearest neighbors. With a sufficiently long time for free diffusion, the individual nanoparticles can sufficiently modify their positions to find the equilibrium lattice positions. A challenge comes from that $\mathrm{Fe}$ is a 
ferromagnetic material. The dipolar magnetic interactions between magnetic nanoparticles increase with the particle volumes and oppose any 2D long range ordering. Previous studies showed that Co nanoparticles larger than $16 \mathrm{~nm}$ tended to form one-dimensional chains and a variety of linear structures [31]. Therefore, in the present case, magnetic interactions play no role in the self-assembling of the 2D densely-packed monolayers of Fe nanoparticles. In fact, magnetization measurements on the Fe nanoparticle deposits display no ferromagnetic hysteresis loops and remnant magnetizations around room temperature, as shown in Fig. 6a, indicating that the Fe nanoparticles are in the superparamagnetic states. It is more likely that attractive van der Waals interactions or dipolar interactions arisen from polarizations dominate the self-assembling of the ordered 2D densely-packed monolayers. In fact, we can also obtain ordered densely-packed monolayers from nanoparticles of nonmagnetic materials. TiN nanoparticles are generated in the gas aggregation cluster source and deposited on the amorphous carbon with similar deposition conditions. From the TEM image shown in Fig. 6b, we can see most of the TiN nanoparticles are involved in a number of ordered monolayers with 2D densely-packing superlattice structures. Similar to the case of Fe nanoparticles, the TiN nanoparticle superlattices can spread over hundreds nm scales.

Regarding the size of the ordered densely-packed 2D monolayer structures that can be achieved with the gas phase cluster deposition, we show in Fig. 7a TEM image of Fe nanoparticle film with a coverage approaching $100 \%$ (i.e., a complete monolayer). By controlling the deposition mass, the densely-packed 2D monolayer structure spreads over the whole substrate surface covered by the deposition spot (at least at the centimeter scale). The monodispersed nanoparticles show a perfect homogeneous distribution in the wide range. Only a few several tens of nanometers sized voids distribute in a very low density. The FFT of the monolayer (inset in Fig. 7) shows two rings of hexagonally arranged spots, related to the first and second orders, attesting to a well-defined hexagonal network ordered over a sufficiently long range. Even though the large scale assembling structure contains domains of $\sim 100 \mathrm{~nm}$ in size, with a number of packing arrays or orientations of the same structure, it is difficult to find any well-defined boundaries between the ordered domains. This result demonstrates the gas phase cluster deposition may provide an efficient way for the fabrication of well-defined patterned superstructures assembled from nanoparticle building blocks on a sufficient large scale.

\section{Conclusion}

We have demonstrated a simple, fast and convenient approach to the fabrication of ordered denselypacked 2D self-assembled monolayer structures of Fe nanoparticles on the template-free surface such as amorphous carbon film by performing gas phase cluster deposition with finely controlled deposition rate and deposition time. This approach has allowed us to prepare 2D superlattice domains composed of well-defined hexagonal nanoparticles networks ordered over lateral dimensions of $100 \sim 200 \mathrm{~nm}$. We have carried out a serial of cluster deposition experiments by carefully varying the nanoparticle deposition dynamics, such as the flux, the lateral migration energy, as well as the migration ability of nanoparticles on the surface. The experimental evidence indicates that the 2D self-assembled monolayer structures are formed by the balance between the diffusion rate of the nanoparticles and their filling speed on the 
surface, which is dependent on the deposition rate. Meanwhile, the attractive interactions between the nanoparticles drive the ordering in the dense-packing arrays. Such mechanism has also allowed us to deposit ordered densely-packed self-assembled monolayer structures of nonmagnetic materials, such as TiN. By controlling the total deposition mass, the densely-packed 2D monolayer domains can spread homogeneously over the whole substrate surface covered by the deposition spot. We believe this method will provide an alternative to the solution chemistry based method that has been commonly used for the fabrication of periodic 2D arrays of thiolate encapsulated nanoparticles from spontaneous organization.

\section{Abbreviations}

TEM:transmission electron microscope;EDX:Energy Dispersive X-ray spectroscopy;XPS:X-ray photoelectron spectroscopy;HRTEM:high-resolution transmission electron microscopy;FFT:fast Fourier transform;RDFs:radial distribution functions.

\section{Declarations}

\section{Supporting information}

The following files are available free of charge.

Size distribution of Fe nanoparticles; The element distribution of Fe nanoparticle arrays; Oxidation state of Fe nanoparticle arrays

\section{Acknowledgment}

The authors gratefully acknowledge the financial support from National Natural Science Foundation of China and the National Key R\&D Program of China.

\section{Authors' contributions}

Dr. C. Liu conceived of the study, and participated in its design andcoordination and drafted the manuscript. Dr. F. Liu supported EDX and XPS analysis. All authors discussed the results and commented on the manuscript. All authors read and approved the fnal manuscript.

\section{Funding}

This research is supported by the National Natural Science Foundation of China (Grant Nos. 11627806, 21875218, 51872261 and U1909214), and the National Key R\&D Program of China (Grant No. 2016YFA0201002).

\section{Availability of Data and Materials}

The datasets used and/or analysed during the current study are available from the corresponding author on reasonable request. 


\section{Competing interests}

The authors declare that they have no competing interests.

\section{References}

1. Bentzon MD, van Wonterghem J, Mørup S, Thölén A,Koch CJW (1989) Ordered aggregates of ultrafine iron oxide particles: 'Super crystals'. Philos Mag B 60: 169-178.

2. Pileni MP (2007) Self-Assembly of Inorganic Nanocrystals: Fabrication and Collective Intrinsic Properties. Accounts Chem Res 40: 685-693.

3. Zhong Z, Gates, Byron, Xia Y,Qin D (2000) Soft Lithographic Approach to the Fabrication of Highly Ordered 2D Arrays of Magnetic Nanoparticles on the Surfaces of Silicon Substrates. Langmuir 16: 10369-10375.

4. Ibanez M, Luo Z, Genc A, Piveteau L, Ortega S, Cadavid D, Dobrozhan O, Liu Y, Nachtegaal M, Zebarjadi M, Arbiol J, Kovalenko MV,Cabot A (2016) High-performance thermoelectric nanocomposites from nanocrystal building blocks. Nat Commun 7: 10766.

5. Bockstaller MR, Kolb R,Thomas EL (2001) Metallodielectric Photonic Crystals Based on Diblock Copolymers. Adv Mater 13: 1783-1786.

6. Courty A, Mermet A, Albouy P, Duval E,Pileni MP (2005) Vibrational coherence of self-organized silver nanocrystals in f.c.c. supra-crystals. Nat Mater 4: 395-398.

7. Kang Y, Ye X, Chen J, Cai Y, Diaz RE, Adzic RR, Stach EA,Murray CB (2013) Design of Pt-Pd Binary Superlattices Exploiting Shape Effects and Synergistic Effects for Oxygen Reduction Reactions. J Am Chem Soc 135: 42-45.

8. Motte L, Billoudet F,Pileni MP (1995) Self-Assembled Monolayer of Nanosized Particles Differing by Their Sizes. J Phys Chem 99: 16425-16429.

9. Weller H (1996) Self-Organized Superlattices of Nanoparticles. Angew Chem 35: 1079-1081.

10. Whetten RL, Khoury JT, Alvarez MM, Murthy S, Vezmar I, Wang ZL, Stephens PW, Cleveland CL, Luedtke WD,Landman U (1996) Nanocrystal gold molecules. Adv Mater 8: 428-433.

11. Mirkin CA, Letsinger RL, Mucic RC,Storhoff JJ (1996) A DNA-based method for rationally assembling nanoparticles into macroscopic materials. Nature 382: 607-609.

12. Macfarlane RJ, Lee B, Jones MR, Harris N, Schatz GC,Mirkin CA (2011) Nanoparticle superlattice engineering with DNA. Science 334: 204-208.

13. Partridge JG, Scott S, Dunbar ADF, Schulze M, Brown SA, Wurl A,Blaikie RJ (2004) Formation of Electrically Conducting Mesoscale Wires Through Self-Assembly of Atomic Clusters. IEEE T Nanotechnol 3: 61-66.

14. Li D, Freitag M, Pearson J, Qiu ZQ,Bader SD (1994) Magnetic phases of ultrathin Fe grown on $\mathrm{Cu}(100)$ as epitaxial wedges. Phys Rev Lett 72: 3112-3115. 
15. Teichert C (2002) Self-organization of nanostructures in semiconductor heteroepitaxy. Phy Rep 365: 335-432.

16. Carroll SJ, Palmer R, Mulheran P, Hobday S,Smith R (1998) Deposition and diffusion of size-selected $\left(\mathrm{Ag}_{400}{ }^{+}\right)$clusters on a stepped graphite surface. Appl Physics A 67: 613-619.

17. Shi Z, Han M, Song F, Zhou J, Wan J,Wang G (2006) Hierarchical self-assembly of silver nanocluster arrays on triblock copolymer templates. J Phys Chem B 110: 18154-18157.

18. Lopes W,Jaeger HM (2001) Hierarchical self-assembly of metal nanostructures on diblock copolymer scaffolds. Nature 414: 735-738.

19. Wegner K, Piseri P, Tafreshi HV,Milani P (2006) Cluster beam deposition: a tool for nanoscale science and technology. J Phys D Appl Phys 39: R439-R459.

20. Binns CW. (2001). Nanoclusters deposited on surfaces. pp 1-49.

21. Jensen $P$ (1999) Growth of nanostructures by cluster deposition: Experiments and simple models. Rev Mod Phys 71: 1695-1735.

22. Bardotti L, Jensen P, Hoareau A, Treilleux M,Cabaud B (1995) Experimental observation of fast diffusion of large antimony clusters on graphite surfaces. Phys Rev Lett 74: 4694-4697.

23. Chen JB, Zhou JF, Häfele A, Yin CR, Kronmüller W, Han M,Haberland H (2005) Morphological studies of nanostructures from directed cluster beam deposition. Eur Phys J D 34: 251-254.

24. Brechignac C, Cahuzac P, Carlier F, Colliex C, Leroux J, Masson A, Yoon B,Landman U (2002) Instability driven fragmentation of nanoscale fractal islands. Phys Rev Lett 88: 196103.

25. Han M, Xu C, Zhu D, Yang L, Zhang J, Chen Y, Ding K, Song F,Wang G (2007) Controllable Synthesis of Two- Dimensional Metal Nanoparticle Arrays with Oriented Size and Number Density Gradients. Adv Mater 19: 2979-2983.

26. Klecha E, Ingert D,Pileni MP (2010) How the Level of Ordering of 2D Nanocrystal Superlattices Is Controlled by Their Deposition Mode. J Phys Chem Lett 1: 1616-1622.

27. Gutiérrez-Wing C, Santiago P, Ascencio JA, Camacho A,José-Yacamán M (2000) Self-assembling of gold nanoparticles in one, two, and three dimensions. Appl Phys A Mater Sci Process 71: 237-243.

28. He L, Chen X, Mu Y, Song F,Han M (2010) Two-dimensional gradient Ag nanoparticle assemblies: multiscale fabrication and SERS applications. Nanotechnology 21: 495601.

29. Wang ZL (1998) Structural Analysis of Self-Assembling Nanocrystal Superlattices. Adv Mater 10: 1330.

30. Murray CB, Kagan CR,Bawendi MG (2000) Synthesis and Characterization of Monodisperse Nanocrystals and Close-Packed Nanocrystal Assemblies. Annu Rev Mater Sci 30: 545-610.

31. Puntes VF (2001) Colloidal Nanocrystal Shape and Size Control: The Case of Cobalt. Science 291: 2115-2117.

\section{Figures}




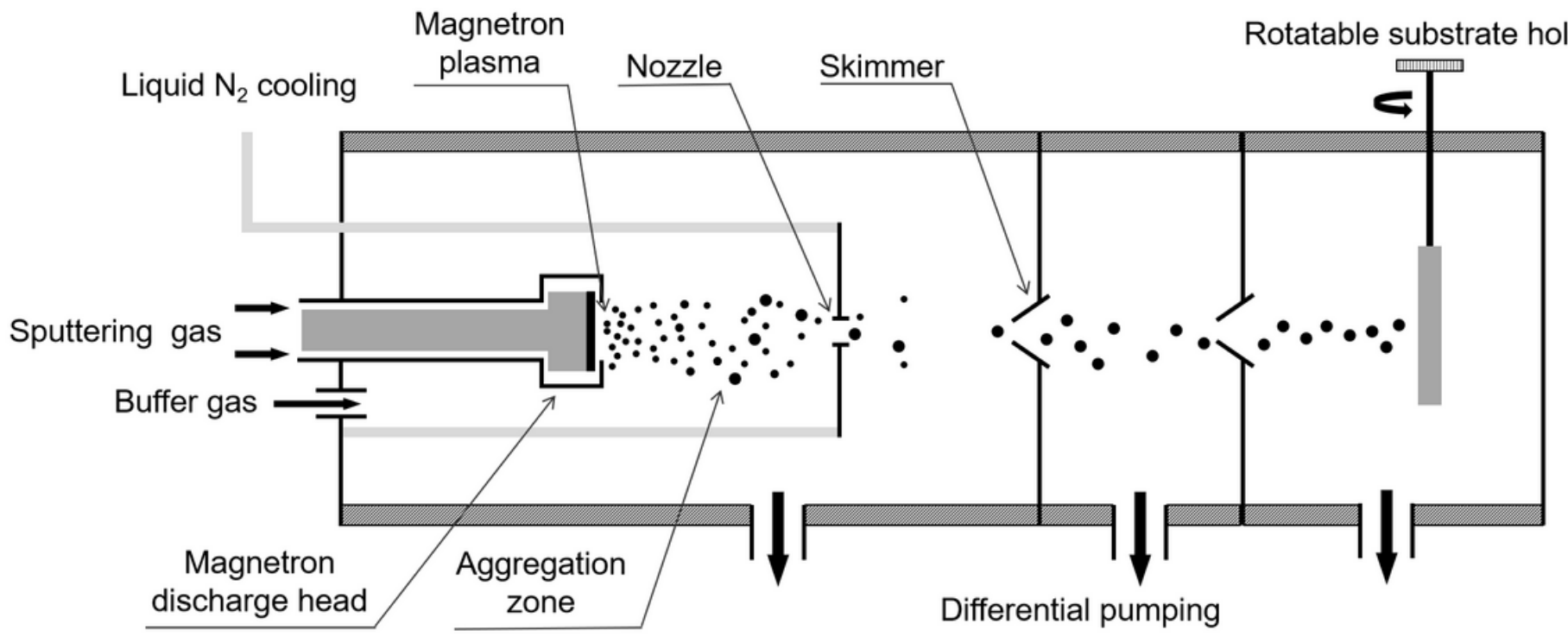

Figure 1

Schematic diagram of a magnetron plasma gas aggregation cluster source and the cluster beam deposition process 

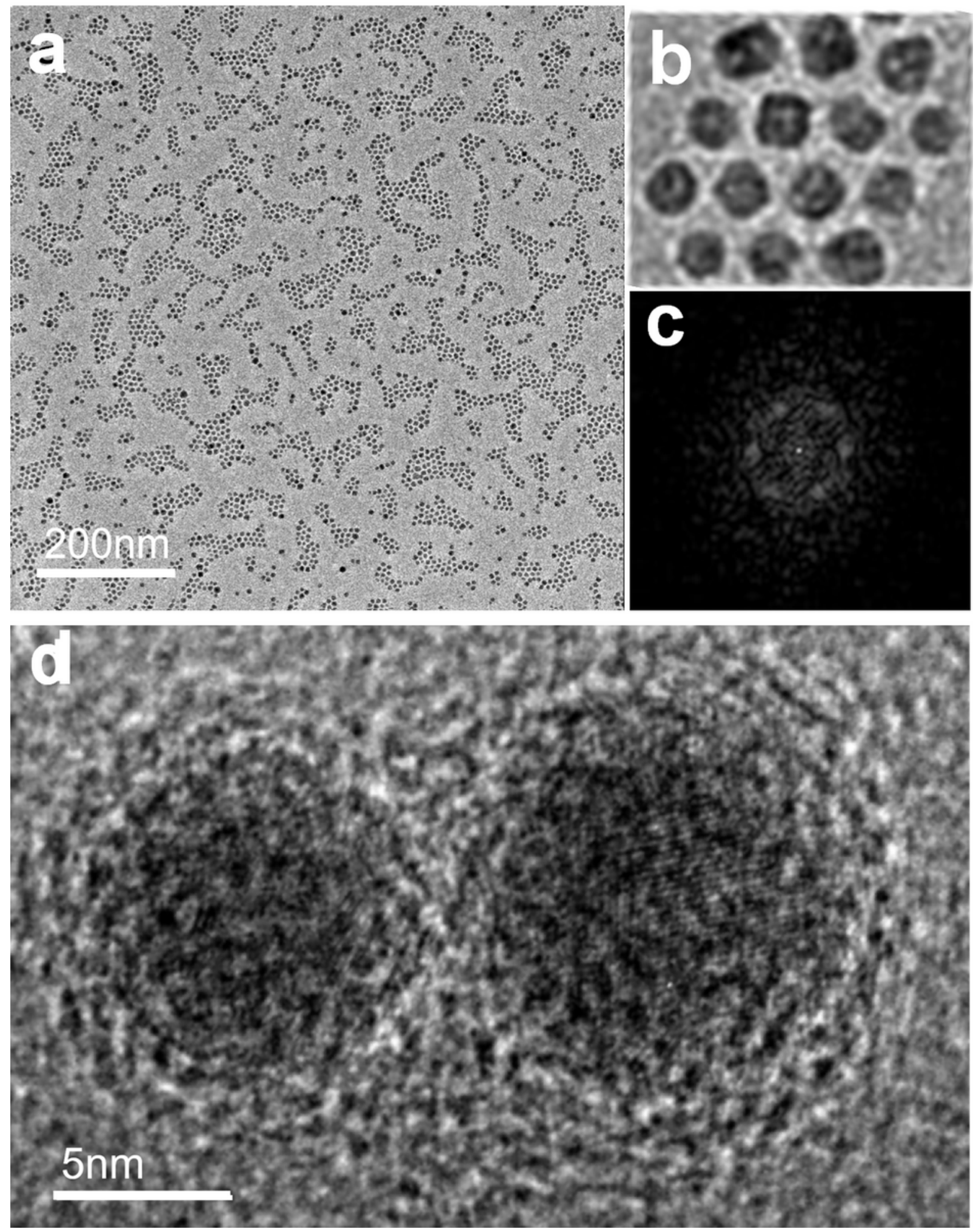

Figure 2

(a) Low magnification TEM image of Fe nanoparticle arrays deposited on a TEM grid covered by amorphous carbon film, with a deposition rate of $0.1 \AA ̊ \cdot s-1$. Domains of close-packed 2D monolayers with long orders can be identified. (b) High magnification TEM image of an ordered densely-packed 2D superlattice of Fe nanoparticles. (c) FFT corresponding to nanoparticle arrays in (a). (d) HTREM image shows lattice images of individual Fe nanoparticles. 


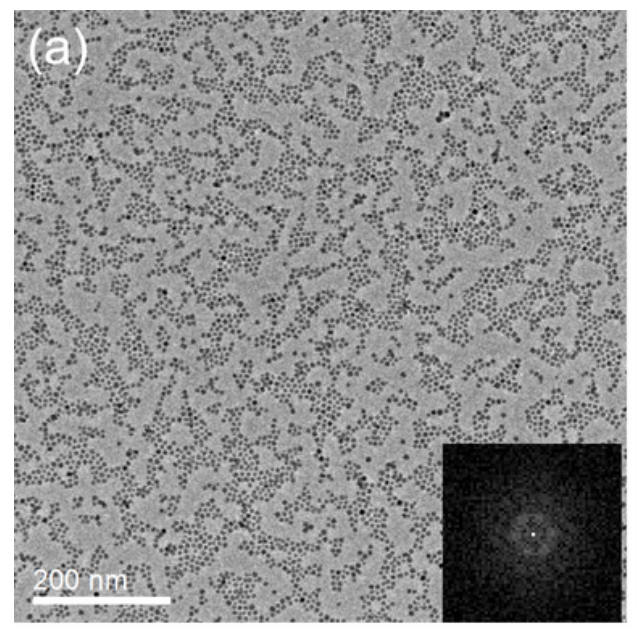

(d)

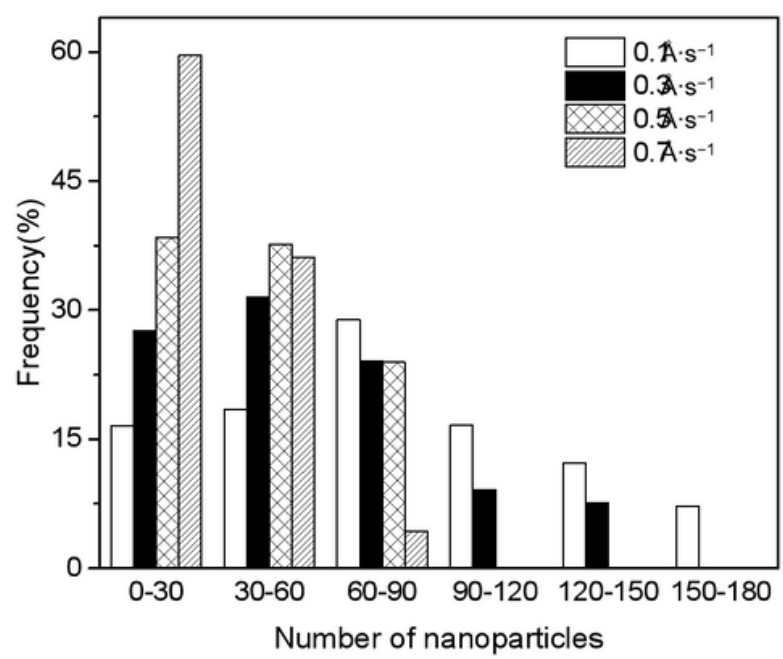

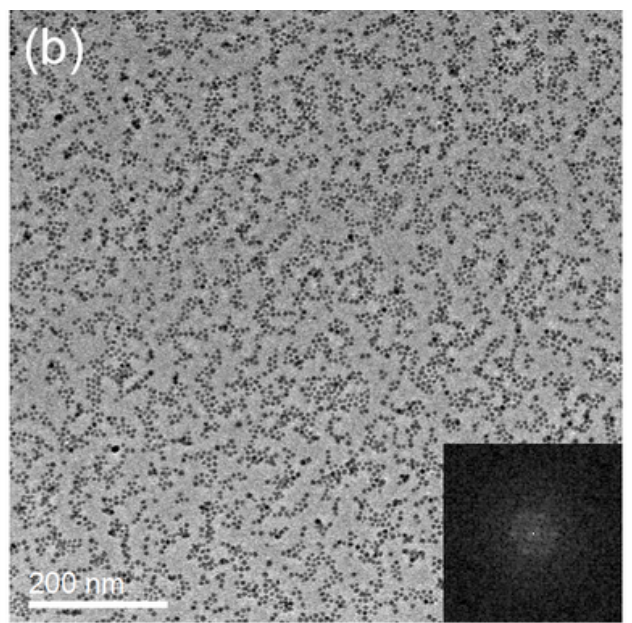

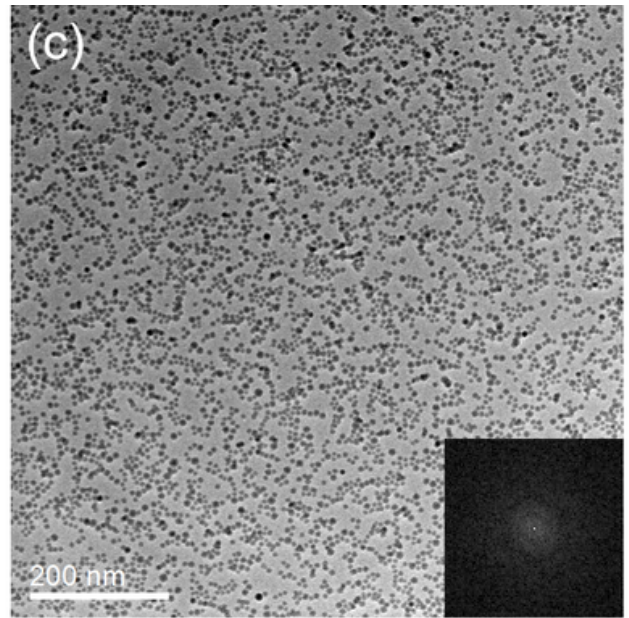

(e)

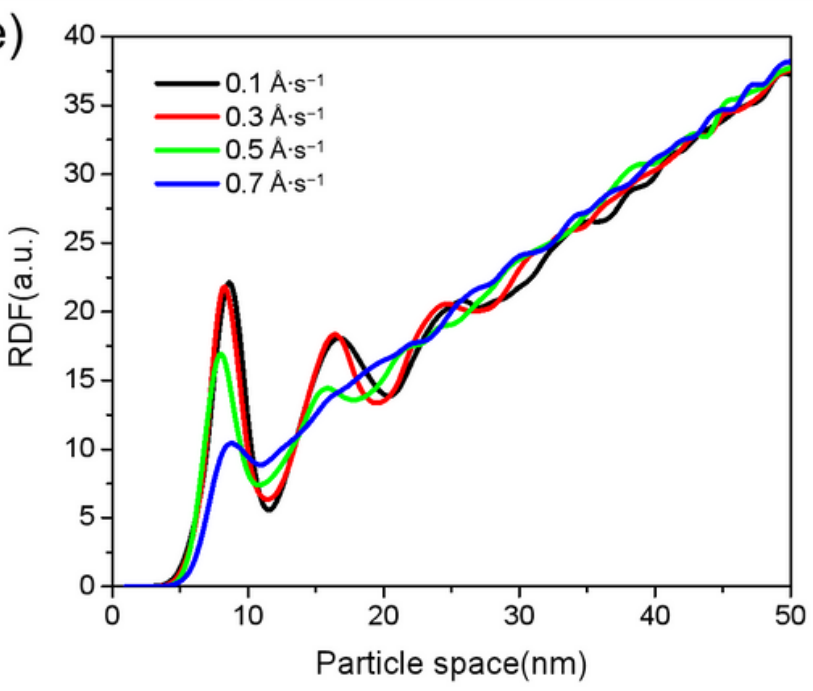

Figure 3

TEM images of Fe nanoparticle arrays with different deposition rate (a) $0.3 \AA ̊ s-1$ (b) $0.5 \AA \bullet s-1$ (c) $0.7 \AA ̊ \cdot s-1$. The insets show the fast Fourier transform of each spatial distribution. (d) Histograms of nanoparticle numbers contained within an individual monolayer domain in the nanoparticle arrays deposited with different deposition rates. The average nanoparticle numbers within an individual monolayer domain are counted to $77,55,39,27$ for deposition rates of $0.1 \AA ̊ \cdot s-1,0.3 \AA \bullet \cdot-1,0.5 \AA \bullet \cdot s-1$, and $0.7 \AA ̊ s-1$, respectively. (e) Radial distribution function of the Fe nanoparticle arrays with different deposition rate. 


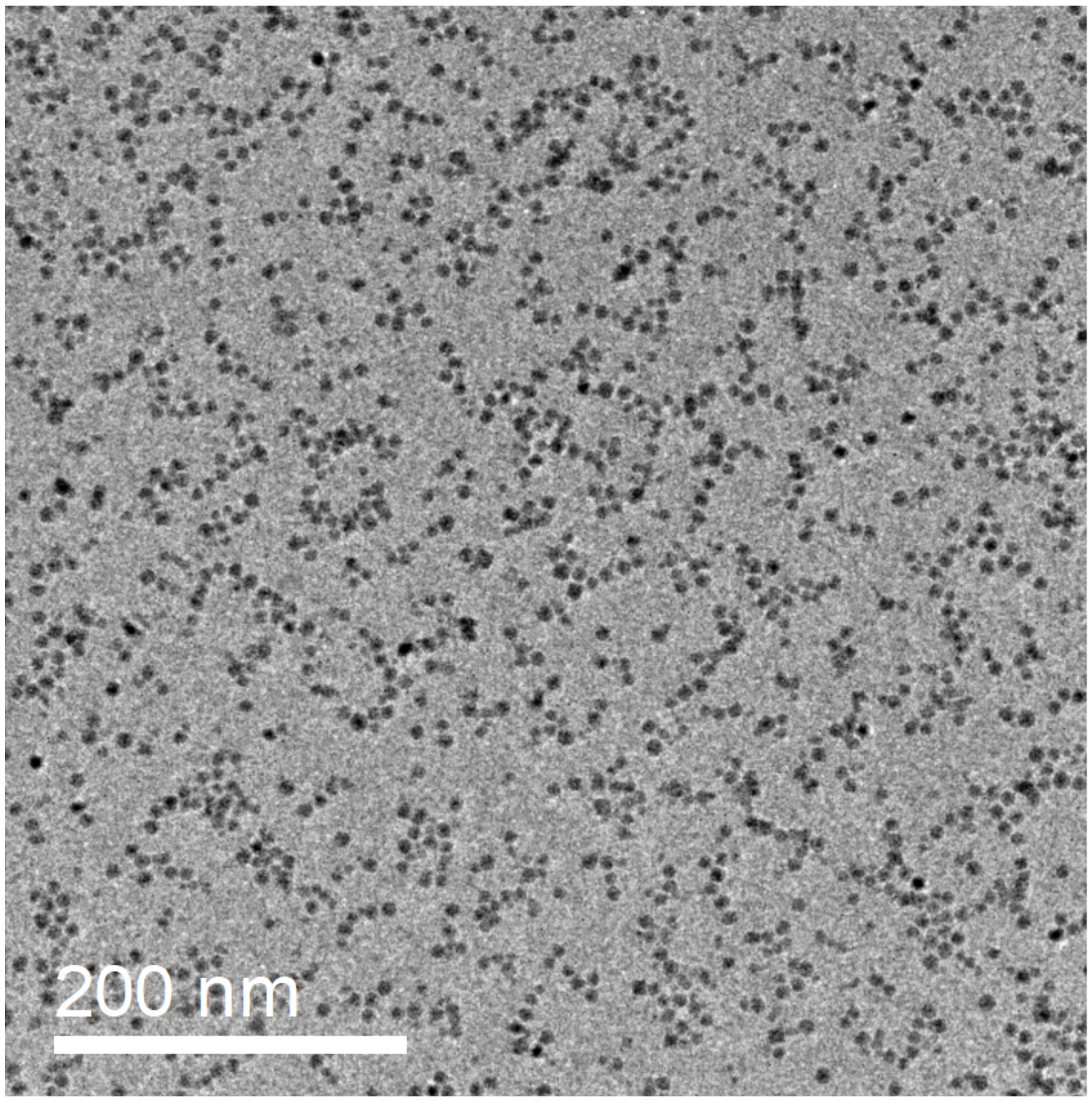

\section{Figure 4}

TEM image of Fe nanoparticle arrays deposited on a Formvar film with a deposition rate of $0.1 \AA \bullet \cdot-1$. 


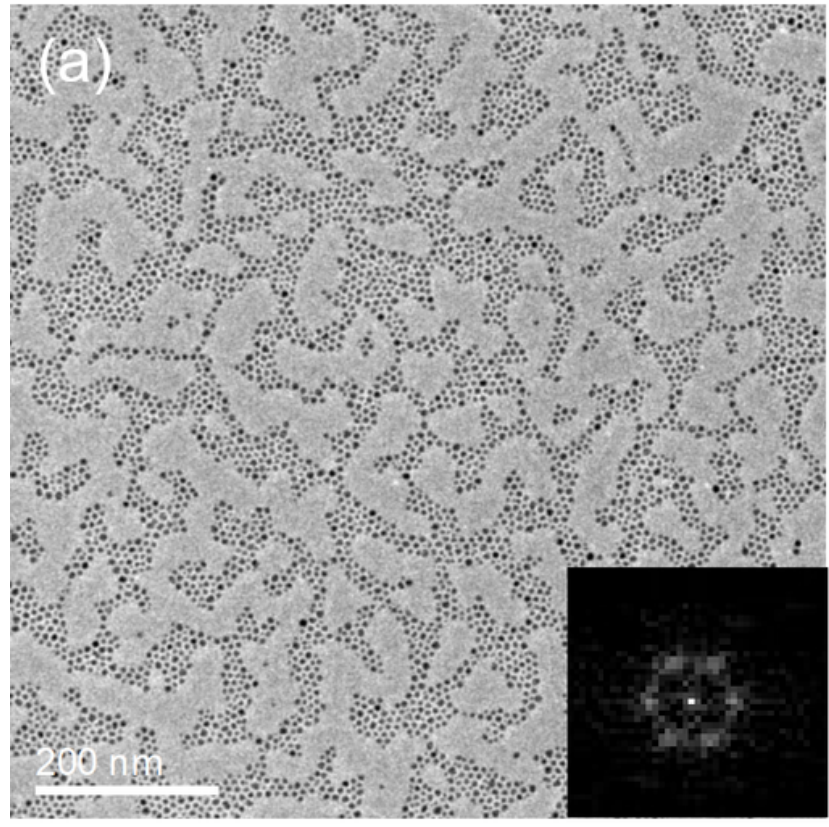

(b)

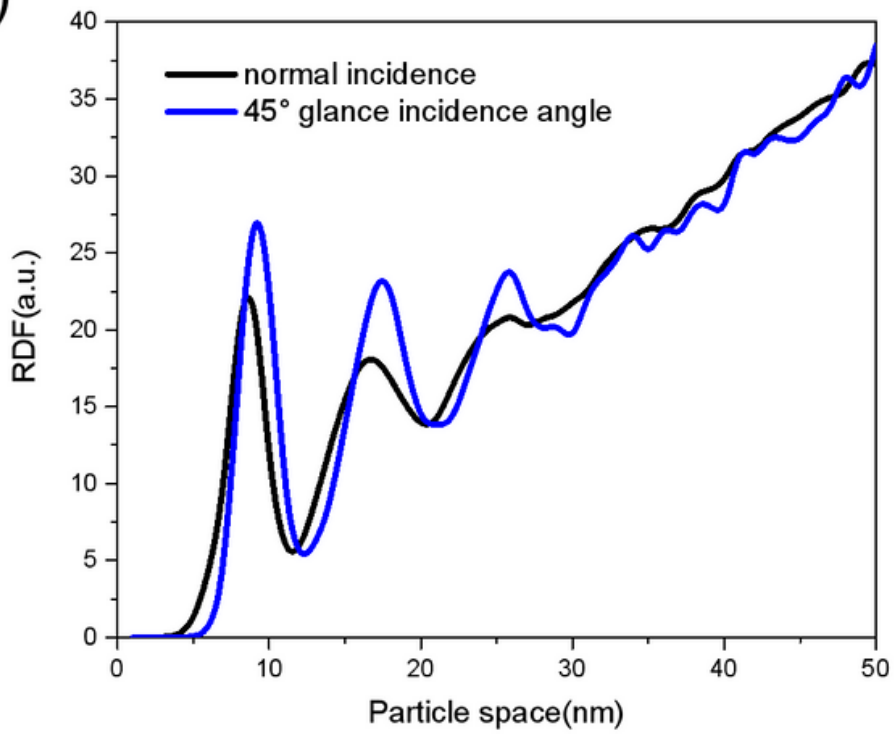

Figure 5

(a) TEM image of Fe nanoparticle arrays deposited with a $45^{\circ}$ glance incidence angle. The inset shows an FFT of the image. (b) A comparison of the RDFs of the Fe nanoparticle arrays deposited with a normal incidence and a $45^{\circ}$ glance incidence.
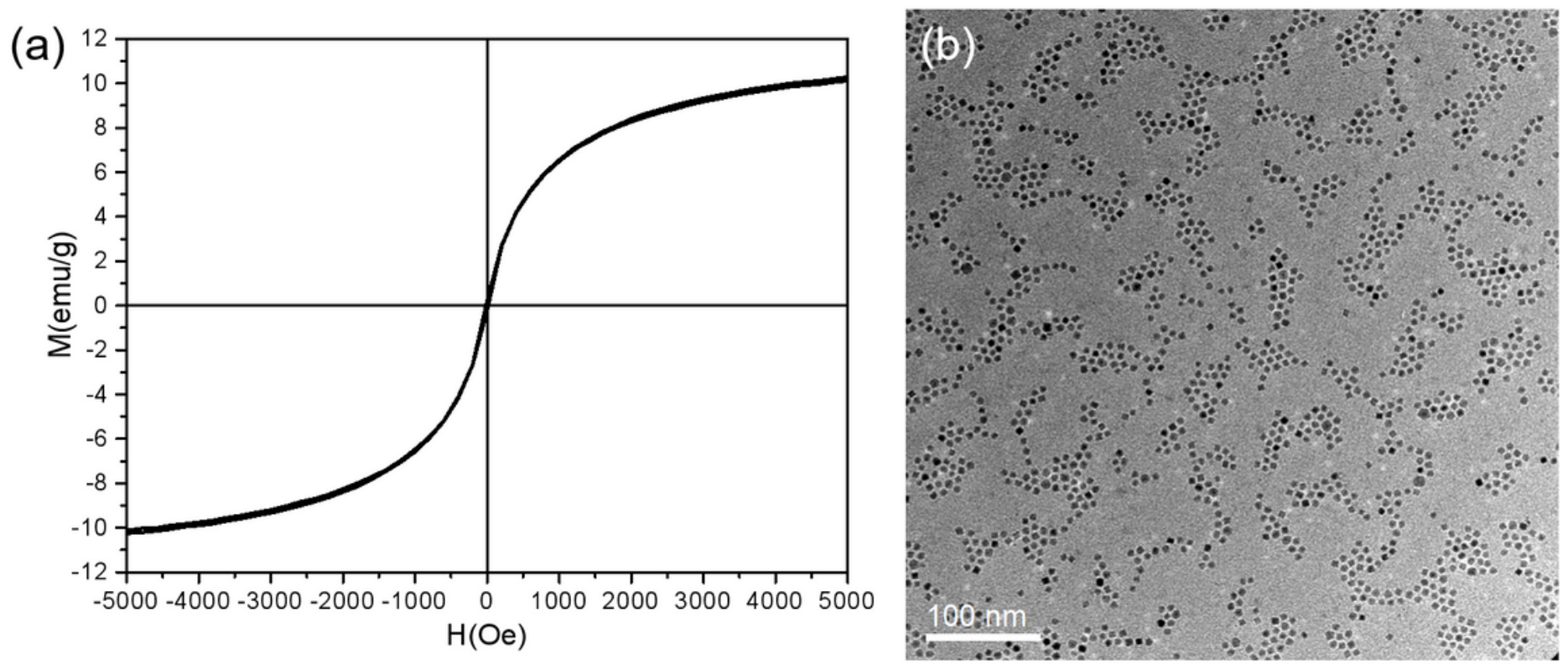

\section{Figure 6}

(a) Hysteresis loop measured at 300K of densely-packed 2D self-assembled Fe nanoparticle arrays, showing that the nanoparticles are in the superparamagnetic states. (b) TEM image of TiN nanoparticle 
arrays deposited on a TEM grid covered by amorphous carbon film, showing that the nanoparticle arrays are composed of domains of ordered densely-packed 2D monolayers.

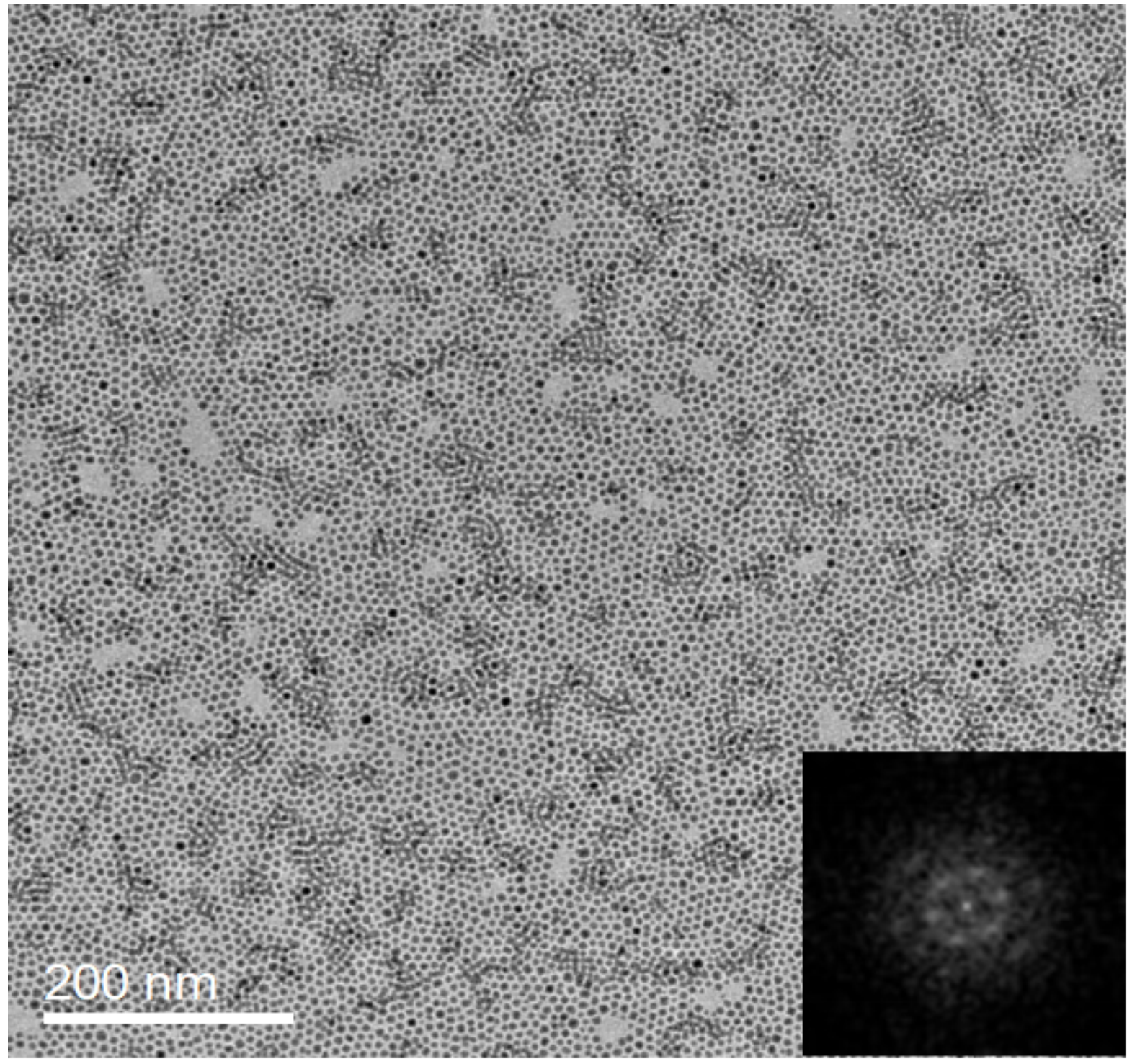

\section{Figure 7}

TEM image of Fe nanoparticle film with nanoparticle coverage approaching $100 \%$. The deposition is performed at an equivalent deposition rate of $0.1 \AA ̊ s-1$ with a $45^{\circ}$ glance incidence. The boundaries between the ordered monolayer domains are hardly identified. The inset show the FFT of the nanoparticle assembling structures.

\section{Supplementary Files}

This is a list of supplementary files associated with this preprint. Click to download.

- supportinginformation.docx 